\title{
The Relationship of SIF between Plate and Plane Fracture Problems and the Effect of the Plate Thickness on SIF
}

\author{
Yongjun Xu and Chuntu Liu \\ Institute of Mechanics, Chinese Academy of Sciences, \\ Beijing 100080, China P.R.
}

Keywords: Eigenfunction, Eigenvalue, Reissner Plate, Stress Intensity Factor

\begin{abstract}
In the present paper, it is shown that the zero series eigenfunctions of Reissner plate cracks/notches fracture problems are analogous to the eigenfunctions of anti-plane and in-plane. The singularity in the double series expression of plate problems only arises in zero series parts. In view of the relationship with eigen-values of anti-plane and in-plane problem, the solution of eigen-values for Reissner plates consists of two parts: anti-plane problem and in-plane problem. As a result the corresponding eigen-values or the corresponding eigen-value solving programs with respect to the anti-plane and in-plane problems can be employed and many aggressive SIF computed methods of plane problems can be employed in the plate. Based on those, the approximate relationship of SIFs between the plate and the plane fracture problems is figured out, and the effect relationship of the plate thickness on SIF is given.
\end{abstract}

\section{INTRODUCTION}

Stress analysis in practical engineering applications inevitably encounters stress singularities caused by sudden changes in geometry, e.g. around re-entrant corners (notches) or, more severely, around crack tips. The complete eigenvalue solution and the stress intensity factor (SIF) calculation play an important role in the linear elasticity fracture mechanics (LEFM). Most of numerical methods, directly or indirectly, make use of the available eigen-solutions when applied to the SIF computation for cracks/notches problems so that singularity can be treated in a more efficient, accurate and reliable way. Recently, $\mathrm{Xu}$ and Yuan [1] presented an effective implementation of the imbedding method for accurate and reliable computation of complete real or complex eigen-solutions in two dimensional notch/crack singularities with multiple materials, arbitrary opening angles and various surface conditions. This algorithm is robust and may be employed by any numerical methods that make use of singular solutions. The singularity causes great difficulty to the numerical solutions that have to be invoked when analytical solutions are not available. A numerical recipe for accurate and efficient computation of stress singularity factors (SIFs) usually consists of two major ingredients, namely a powerful numerical method for the general stress 
analysis and a novel approach to obtaining the desired SIFs which may include special treatment of various singularities. The underlying numerical method employed in this paper is the finite element method of lines (FEMOL) [2-5], which is a newly developed, general-purpose, semi-analytical method. In this method, a partial differential equation defined on an arbitrary domain is semidiscretized, by finite element techniques via energy theorems or variational principles, into a system of ordinary differential equations (ODEs) defined on straight or curved mesh lines. Recently, Xu and Yuan [6] exploited the orthogonal relationship between eigen-functions for anti-plane and inplane notch problems, and a set of contour integral extraction methods (so called orthogonal integral extraction method) for SIF calculation is developed, which appears to be most powerful and efficient. Xu [7] extended the orthogonal integral extraction method to the general two-dimensional fracture problems. In the present paper, It is shown that the zero series eigenfunctions of Reissner plate cracks/notches fracture problems are analogous to the eigenfunctions of anti-plane and inplane. The singularity in the double series expression of plate problems only arises in zero series parts. In view of the relationship with eigen-values of anti-plane and in-plane problem, the solution of eigen-values for Reissner plates consists of two parts: anti-plane problem and in-plane problems. As a result, the corresponding eigenvalues or the corresponding eigenvalue solving programs with respect to the anti-plane and in-plane problems can be employed and many aggressive SIF computed methods of plane problems can be employed in the plate problems. Based on those, the approximate relationship of SIFs between the plate and the plane fracture problems is figured out, and the effect relationship of the plate thickness on SIF is given. Two examples are given to show the two approximate relationships.

\section{PRELIMINARY CONSIDERATION}

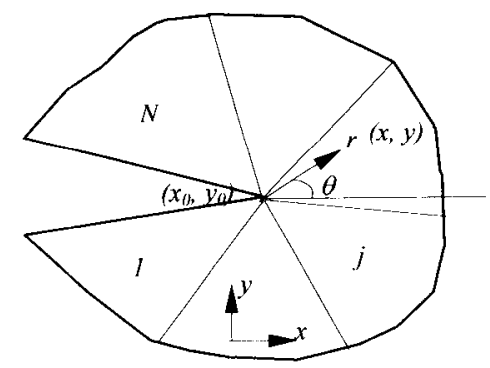

Fig. $1 \mathrm{~N}$-material notch problem

Figure 1 shows a two dimensional singular problem with $N$ different materials around notch/crack tip. The body forces are assumed to be negligible. For convenience sake we omit the different material domain subscript $j$ shown in the Fig. 1. In the polar coordinates shown in Fig. 1. for the Reissner plate, the general displacement components can be written as the following double series form

$$
w^{\prime}=\sum_{i} \sum_{n} r^{\lambda_{1}+n} c_{n i}^{\prime}(\theta),\left\{\begin{array}{l}
\psi_{r}^{\prime}=\sum_{i} \sum_{n} r^{\lambda_{1}+n} a_{n i}^{\prime}(\theta) \\
\psi_{\theta}^{j}=\sum_{i} \sum_{n} r^{\lambda_{i}+n} b_{m i}^{\prime}(\theta)
\end{array}\right.
$$

It can be showed that the singularity maybe appear when $n=0$ and in the first two or three 
terms of $i$. Here we only take account of the main part of $n=0$, the Reissner plate could be divided into two basic parts of anti-plane part and in-plane part. So the well-known Williams' displacement $w_{0}(r, \theta)$ and potential function $\phi_{0}(r, \theta)$ are

$$
\text { (i) } w_{0}(r, \theta)=\sum_{i, 0,1,2,3} \alpha_{i} r^{\lambda_{i}} f_{i}(\theta) \quad \text { (ii) } \phi_{0}(r, \theta)=\sum_{i=0.1,2, \ldots} \alpha, r^{\lambda_{i}+1} F_{i}(\theta)
$$

where both $f_{i}(\theta)$ and $F_{i}(\theta)$ are certain normalization eigen-functions that corresponding to the eigenvalue $\lambda_{t}$, and the well known explicit form is

$$
\begin{aligned}
& f_{i}(\theta)=A_{1} \cos \lambda_{i} \theta+B_{i} \sin \lambda_{i} \theta \\
& F_{i}(\theta)=A_{i} \sin \left(\lambda_{i}+1\right) \theta+B_{i} \cos \left(\lambda_{1}+1\right) \theta+C_{i} \sin \left(\lambda_{i}-1\right) \theta+D_{i} \cos \left(\lambda_{i}-1\right) \theta
\end{aligned}
$$

where $\left\{A_{i}, B_{i}\right\}$ and $\lambda_{i}$ in $f_{i}(\theta)$ are real constants, $\left\{A_{i}, B_{i}, C_{i}, D_{i}\right\}$ and $\lambda_{i}$ in $F_{i}(\theta)$ are complex constants. The corresponding displacement $\left(\psi_{r 0,}, \psi_{00_{0}}\right)$ for plane part can be derived from the stress potential function

$$
\psi_{r(1, r}=r^{\lambda} \frac{\left(1-v \lambda_{t}\right)\left(1+\lambda_{i}\right) F_{1}(\theta)+F_{t}(\theta)}{\lambda E} \quad \psi_{\theta 0 t}=r^{i} \frac{\left(1+\lambda_{1}-\left(1-\lambda_{1}\right) \lambda_{i} v+2 \lambda_{i}^{2}\right) F_{i}^{\prime}(\theta)+F_{i}(\theta)}{\lambda(1-\lambda) E}
$$

The corresponding stress components of both anti-plane and plane problems are

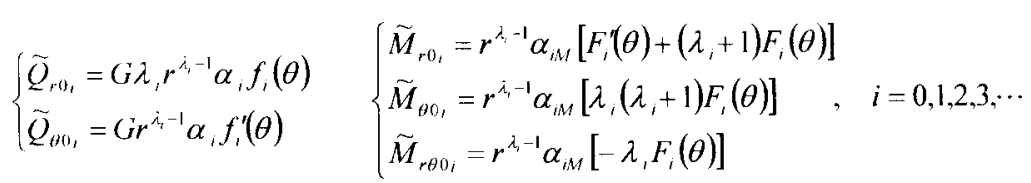

When $r \rightarrow 0$, the stress in formula (5) will tend to infinity because there are singular terms $r^{\lambda_{1}} 1$ in the expansions. Compared the anti-plane part and the in-plane part of Reissner plate problem shown in equations (2-5) with real anti-plane and in-plane problems ${ }^{|6|}$, the following assimilated relationships can be deduced

$$
\begin{aligned}
& w_{0} \sim w, \quad \widetilde{Q}_{r 0} \sim \tau_{r=}, \quad \widetilde{Q}_{\theta 0} \sim \tau_{\theta z} \\
& \left\{\begin{array}{l}
\psi_{r \theta} \sim u_{r}, \quad \psi_{\theta 0} \sim u_{\theta} \\
\tilde{M}_{r 0} \sim \sigma_{r r}, \quad \tilde{M}_{\theta 0} \sim \sigma_{\theta \theta}, \quad \tilde{M}_{r \theta 0} \sim \sigma_{r \theta}
\end{array}\right.
\end{aligned}
$$

where $\left\{w, \tau_{r=}, \tau_{t \varepsilon}\right\}$ and $\left\{u_{r}, u_{\theta}, \sigma_{r r}, \sigma_{\theta \theta}, \sigma_{r \theta}\right\}$ are the displacements and stresses corresponding to the anti-plane and in-plane problems, respectively.

\section{GENERAL DEFINITION OF SINGULARITY INTENSITY FACTORS (SIFs)}

For two dimensional fracture problems, the general singularity intensity factors defined as

$$
K_{\mathrm{III}}=\lim _{r \rightarrow 0}\left(\sqrt{2 \pi} r^{1-\lambda} \max _{\theta}\left(\tau_{r \theta \theta}(r, \theta)\right)\right),\left\{\begin{array}{l}
K_{\mathrm{I}}=\lim _{r \rightarrow 0}\left(\sqrt{2 \pi} r^{1-\lambda} \max _{\theta}\left(\sigma_{\theta 0}(r, \theta)\right)\right) \\
K_{\mathrm{II}}=\lim _{r \rightarrow 0}\left(\sqrt{2 \pi} r^{1-\lambda} \max _{\theta}\left(\sigma_{r \theta \theta}(r, \theta)\right)\right)
\end{array}\right.
$$

For Reissner plate fracture problems, substitute the shears and moments into Eq. (7), the SIFs are generally defined as 


$$
K_{\mathrm{III}} \approx \lim _{r \rightarrow 11}\left(\sqrt{2 \pi} r^{1-\lambda} \max _{\theta}\left(\frac{5}{4}\left(1-\zeta^{2}\right) \widetilde{Q}_{\theta 0}(r, \theta)\right)\right),\left\{\begin{array}{l}
K_{1} \approx-\lim _{r \rightarrow 0}\left(\sqrt{2 \pi} r^{1-\lambda} \max _{\theta}\left(\frac{\dot{h}}{2} \zeta \widetilde{M}_{\theta 0}(r, \theta)\right)\right) \\
K_{\mathrm{II}} \approx-\lim _{r \rightarrow 0}\left(\sqrt{2 \pi} r^{1-\lambda} \max _{\theta}\left(\frac{h}{2} \zeta \widetilde{M}_{r \theta 0}(r, \theta)\right)\right)
\end{array}\right.
$$

where $\zeta=2 z / h$, we take the maximum value as $\zeta=1, \lambda$ is the corresponding eigenvalue.

\section{TWO RELATIONSHIPS OF SINGULARITY INTENSITY FACTORS (SIFs)}

Firstly, we consider the different between zero order stresses $\left\{\check{M}_{0}, \breve{Q}_{0}\right\}$ and the real stresses $\{M . Q\}$; secondly, compare the equivalent between the Reissner plate and the plane problems; we consider the same equivalent boundary conditions between the Reissner plate and the plane problems. We have the following approximate relationship with respect to SIFs between the Reissner plate and the plane fracture problems

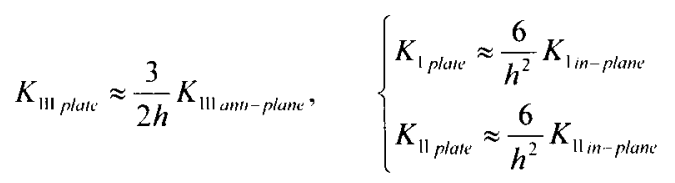

For the two Reissner plate notch/crack problems, the only different part is the plate thickness $\left(h_{1}\right.$ and $h_{2}$ ). Form Eq. (9), the approximate relationship with respect to SIFs between the different $\mathrm{pl}$ ate thickness can be deduced

$$
\frac{K_{\mathrm{III}}\left(h_{1}\right)}{K_{\mathrm{III}}\left(h_{2}\right)} \approx \frac{h_{2}}{h_{1}}, \quad \frac{K_{1 . \mathrm{II}}\left(h_{1}\right)}{K_{\mathrm{l.11}}\left(h_{2}\right)} \approx\left(\frac{h_{2}}{h_{1}}\right)^{2}
$$

\section{NUMERICAL EXAMPLES}

To assess the performance of the two relationships, a number of numerical examples are given in this section. The following examples are computed by the newly developed method [6-7] namely orthogonal integral extraction method.

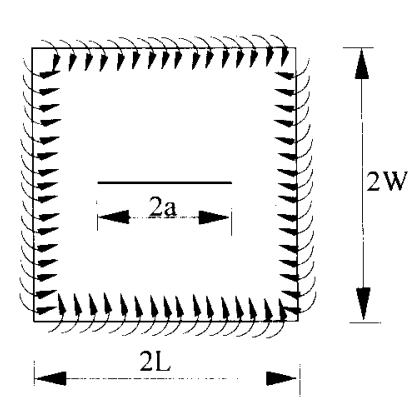

\section{$\Lambda \Lambda \Lambda \Lambda \Lambda \Lambda \Lambda \Lambda \Lambda$}

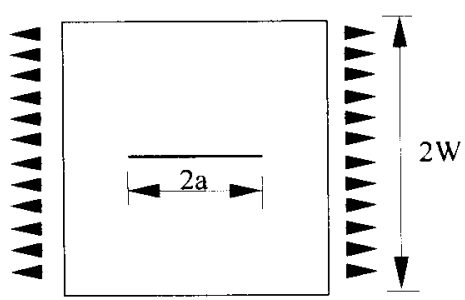

\section{MTINTHT}

$2 \mathrm{~L}$

(a) Bending plate with a center crack

(b) Uniform stretch with a center crack

Fig. 2 Finite plate with a center crack 
Example 1 Approximate relationship of SIFs between bending plate and uniform stretch plane

In this example, as Fig. 2 shows, we consider a finite bending plate with a center crack and a finite uniform stretch plane with a center crack. The crack length is $2 a$, the plate thickness is $h$, and the necessary constants are $2 L=2 W=4 a, E=2 \times 10^{6}, v=0.3, a=1, h=1$. Table 1 gives a list of the computed results of SIFs $K_{1}$ and the approximate ratio of the SIFs for bending plate and plane. Where $r_{0}$ indicates the integral radius along the crack tips.

Table 1 . The computed results of finite plate with a center crack

\begin{tabular}{cccc}
\hline$r_{0}$ & Bending & Uniform stretch & $K_{\text {lplate }} / K_{\text {lplane }}$ (Theory $\left.\approx 6\right)$ \\
\hline 0.00001 & 9.728794 & 2.363948 & 4.115486 \\
0.0001 & 9.743342 & 2.363946 & 4.121643 \\
0.001 & 9.784121 & 2.363943 & 4.138899 \\
0.01 & 9.865933 & 2.363946 & 4.173502 \\
0.1 & 9.832484 & 2.363945 & 4.159354 \\
0.5 & 10.099617 & 2.363951 & 4.272346 \\
\hline
\end{tabular}

Example 2 Approximate effect on SIFs $\left(K_{1}\right)$ of the different plate thickness

In this example, as Fig. 2(a) shows, we consider a finite bending plate with a center crack. The crack length is $2 a$, and the necessary constants are $2 L=2 W=4 a, E=2 \times 10^{6}, v=0.3, a=1$. The three cases of the different thickness are $h_{1} / a=0.1, h_{2} / a=0.5$ and $h_{3} / a=1.0$. Table 2 gives a list of the computed results of SIFs $K_{l}$, where $r_{0}$ indicates the integral radius along the crack tips. Fig. 3 gives out two ratio curves of the different SIFs $K_{I}$ with respect to the different cases, the approximate values match very well with the theory values.

\begin{tabular}{c|ccc}
\multicolumn{3}{c}{ Table 2. The computed results of $K_{1}$ for finite bending plate with a center crack } \\
\hline$h / a$ & 0.1 & 0.5 & 1.0 \\
\hline 0.00001 & 793.823490 & 36.101453 & 9.728794 \\
0.0001 & 793.800340 & 38.376926 & 9.743342 \\
0.001 & 794.154503 & 42.004670 & 9.784121 \\
0.01 & 800.501913 & 33.544143 & 9.865933 \\
0.1 & 842.029973 & 35.785873 & 9.832484 \\
0.5 & 929.598925 & 38.401774 & 10.099617 \\
\hline
\end{tabular}

\section{CONCLUDING REMARKS}

In this present paper, the Relationship of SIF between plate and plane fracture problems and the effect of the plate thickness on SIFs are discussed. Both of the two approximate relationships are checked by two simply examples. From example 2 we know that the numerical values match very well with the theory values in the second relationship with respect to the different plate thickness. From the two relationships, we can give an approximate estimate for the unsolved problems from 
the corresponding solved problems and a check to computed results and some methods based on the well known examples.

\section{Acknowledgments}

This work was supported by the National Natural Science Foundation of China.

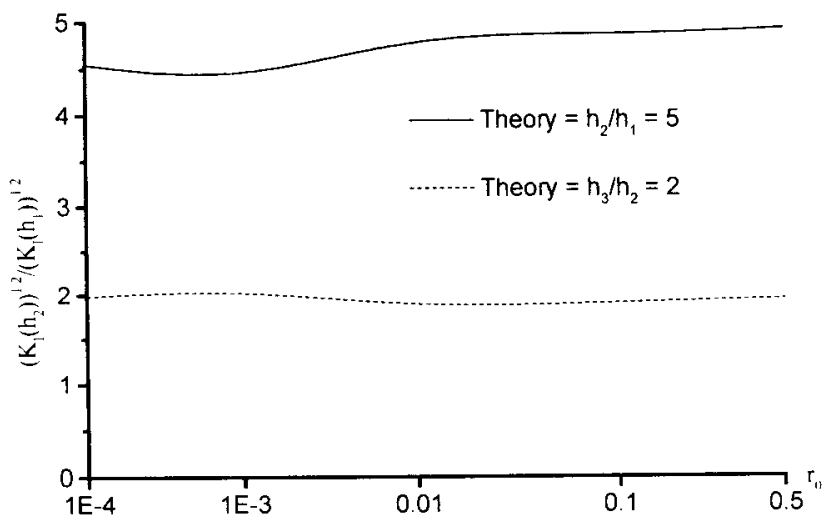

Fig. $3 \sqrt{\frac{K_{1}\left(h_{2}\right)}{K_{1}\left(h_{1}\right)}}$

\section{REFERENCES}

1. Yongjun Xu and Si Yuan, International Journal of Fracture, Vol. 18, (1996) p. 373-381.

2. Si Yuan, Proceedings of 1st National Conference on Analytical and Numerical Combined Methods, Hunan (May, 1990) p. 132-136 (in Chinese).

3. Si Yuan and Jianling Gao, Proceedings of International Conference on EPMESC, Macau, 3 (Aug. 1-3, 1990) p. 517-526.

4. Si Yuan, Chinese Journal of Numerical Mathematics and Applications, 15, 1 (1993) p. 45-59.

5. Si Yuan, The Finite Element Method of Lines: Theory and Applications, Science Press, Beijing,New York (1993).

6. Yongjun $\mathrm{Xu}$, Complete eigenvalue solution and SIF calculation in fracture mechanics based on FEMOL, Ph.D. Thesis. Department of Civil Engineering, Beijing: Tsinghua University, 1996 (in Chinese)

7. Yongjun Xu, SIF calculation in plates, shells and three dimensional problems based on FEMOL, Postdoctoral report. Institute of Mechanics, Beijing: Chinese Academy of Sciences, 1999 (in Chinese) 
Fracture and Strength of Solids IV

10.4028/www.scientific.net/KEM.183-187

The Relationship of SIF between Plate and Plane Fracture Problems and the Effect of the Plate Thickness on SIF

10.4028/www.scientific.net/KEM.183-187.163

\section{DOI References}

[] 5 ig.3 /K(h, (h) EFERENCES . Yongjun Xu and Si Yuan, International Journal of Fracture, Vol. 18, (1996) p. 373-381.

doi:10.1007/BF00012429 